\title{
Harmonic Suppressed Dual-Band Bandpass Filter with Independently Tunable Center Frequencies and Bandwidths
}

\author{
Girdhari Chaudhary ${ }^{1} \cdot$ Yongchae Jeong ${ }^{1, *} \cdot{\text { Jongsik } \text { Lim }^{2}}^{2}$
}

\begin{abstract}
This paper presented a novel approach for the design of a tunable dual-band bandpass filter (BPF) with independently tunable passband center frequencies and bandwidths. The newly proposed dual-band filter principally comprised two dual-mode single band filters using common input/output lines. Each single BPF was realized using a varactorloaded transmission line resonator. To suppress the harmonics over a broad bandwidth, a defected ground structure was used at the input/output feeding lines. From the experimental results, it was found that the proposed filter exhibited the first passband center frequency tunable range from 1.48 to $1.8 \mathrm{GHz}$ with a 3-dB fractional bandwidth (FBW) variation from $5.76 \%$ to $8.55 \%$, while the second passband center's frequency tunable range was 2.40 to 2.88 $\mathrm{GHz}$ with a 3-dB FBW variation from $8.28 \%$ to $12.42 \%$. The measured results of the proposed filters showed a rejection level of $19 \mathrm{~dB}$ up to more than 10 times the highest center frequency of the first passband.
\end{abstract}

Key Words: Defected Ground Structure, Harmonic Suppression, Independently Tunable Center Frequency and Bandwidths, Tunable Dual-Band Bandpass Filter, Varactor Diode.

\section{Introduction}

Various wireless communication standards, such as wireless local area networks (WLANs), worldwide interoperability for microwave access (WiMAX), wideband code division multiple access (W-CDMA), long-term evolution (LTE), etc., have been developed throughout the world and more standards will likely emerge in the near future. The integration of separate standards into one unit increases the size, cost, and complexity of wireless systems. The need for the design of low-cost, compact robust radio frequency components such as tunable bandpass filters (BPFs) operating at multiple frequency bands to meet the demands of next generation wireless systems have become apparent.

In some applications of wireless communication systems, radios themselves sense the available spectrum and decide on the communications channel (frequency, bandwidth, and modulation scheme) to be used. Therefore, it is important to design a multi-band BPF with a tunable center frequency and bandwidths. To meet these requirements, various approaches have been applied to design tunable BPFs using different kinds of tuning de- vices, such as micro-electromechanical system (MEMS) devices [1, 2], ferroelectric devices/capacitors [3], PIN diodes [4], and semiconductor varactor diodes [5-9]. These tuning devices allow tunability of the center frequency of filters by changing the effective length of their resonators and the variation of the bandwidth by changing coupling between resonators.

Semiconductor varactors are widely used in designing tunable BPFs due to high tuning speed and reliability [5-9]. In comparison to the design of single-band tunable filters, only a limited number of studies have been undertaken to design filters with a tunable center frequency and bandwidth independent of one another [10, 11]. There have been some attempts to design tunable dual-passband filters [12, 13]. However, no studies have addressed the design of a tunable dual-band BPF with independently tunable center frequencies and bandwidths.

With the tunable passband frequency, the harmonic bands that degrade the out-of-passband characteristics are also tuned. Thus, the suppression of harmonics is one of the critical issues related to tunable filters. This issue has rarely been addressed in the design of tunable du-

Manuscript received January 2, 2013 ; Revised March 5, 2013 ; Accepted March 26, 2013. (ID No. 20130102-001J)

${ }^{1}$ Department of Electronics and Information Engineering, Chonbuk National University, Jeonju, Korea.

${ }^{2}$ Department of Electrical and Communication Engineering, Soonchunhyang University, Asan, Korea.

${ }^{*}$ Corresponding Author : Yongchae Jeong (e-mail : ycjeong@jbnu.ac.kr)

This is an Open-Access article distributed under the terms of the Creative Commons Attribution Non-Commercial License (http://creativecommons.org/licenses/ by-nc/3.0) which permits unrestricted non-commercial use, distribution, and reproduction in any medium, provided the original work is properly cited. 
al-band filters.

In this paper, dual-band BPFs with independently tunable passband center frequencies and bandwidths are presented. From theoretical even- and odd-mode analysis, it is found that the passband center frequencies can be varied by controlling the odd- and even-mode resonant frequencies. The separation between the odd- and even-mode resonant frequencies is proportional to the bandwidths of passbands; these can be controlled independently by the even-mode resonant frequencies by keeping the odd-mode resonant frequencies constant. To suppress the harmonics, the defected ground structure (DGS) is used at the input/output feeding lines. The band-rejection characteristics of the DGS are utilized to suppress the harmonics of the proposed BPF.

\section{Design and Implementation}

Fig. 1(a) shows the proposed two-pole varactor-tuned dual-band BPF. It consists of two single-band BPFs combined with common input and output ports. Each single-band BPF comprises dual-mode resonators that con-

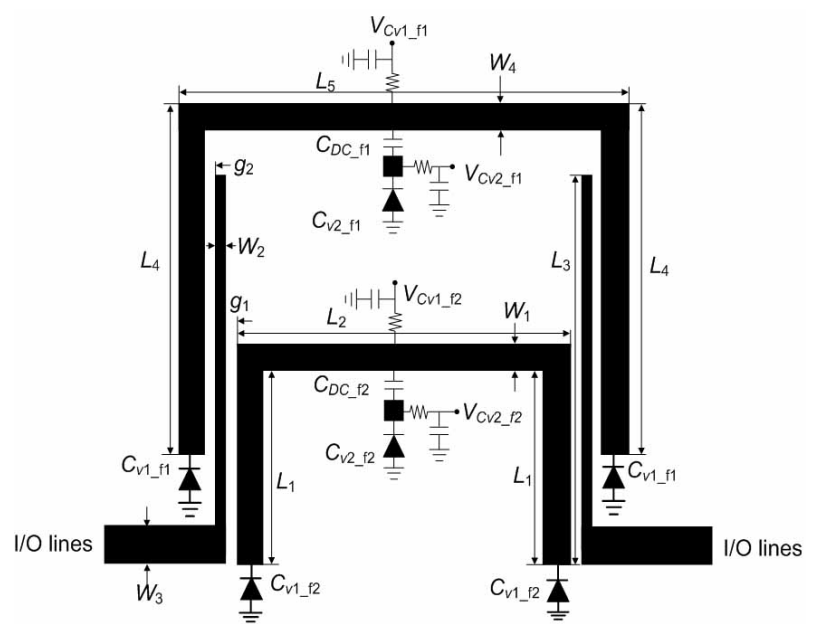

(a)

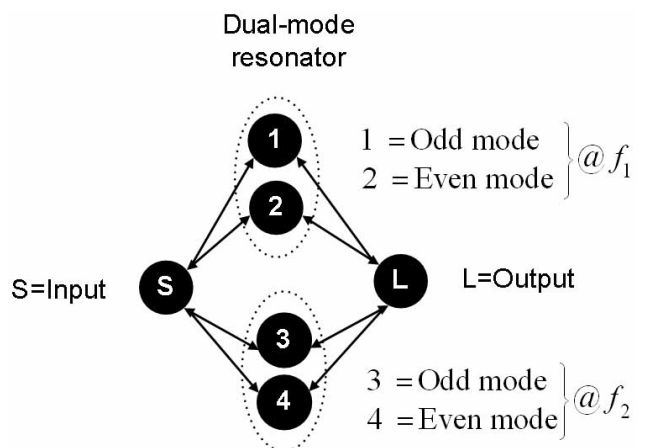

(b)

Fig. 1. (a) Layout of the proposed two-pole dual-band tunable filter I and (b) its coupling scheme.

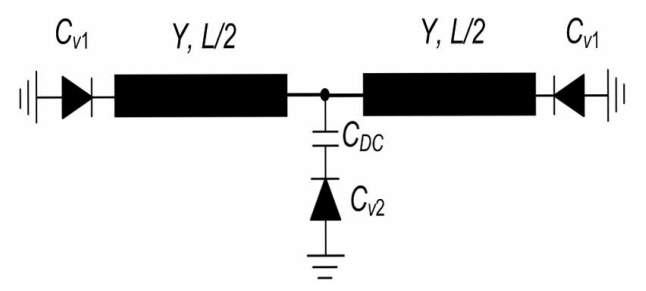

(a)

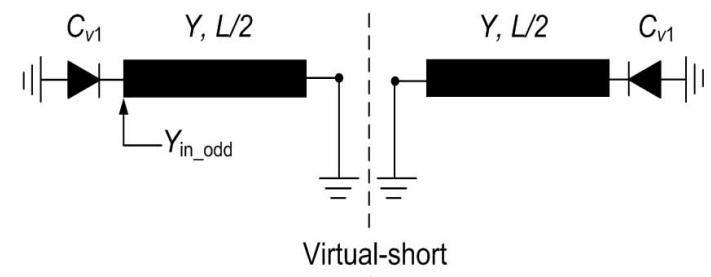

(b)

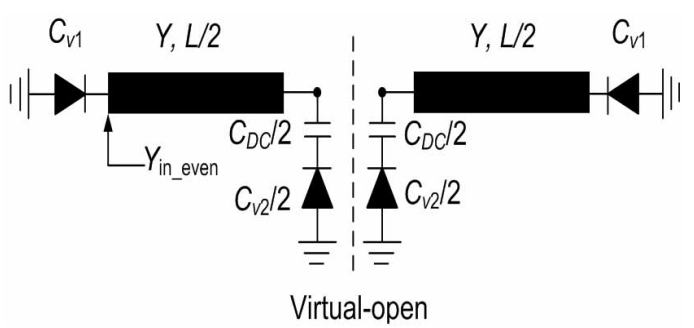

(c)

Fig. 2. (a) Basic structure of the proposed resonator, (b) oddmode excitation equivalent circuit, and (c) even-mode excitation equivalent circuit.

tain a transmission line and three varactor diodes. The coupling scheme of the proposed filter is shown in Fig. 1(b), where $S$ and $L$ denote input and output ports. Nodes 1 and 3 denote the odd modes and nodes 2 and 4 denote the even modes at the first passband $\left(f_{1}\right)$ and second passband $\left(f_{2}\right)$ center frequencies, respectively. Since these two operating modes do not couple to each other, a simple tuning scheme can be obtained.

Fig. 2(a) shows the basic structure of the proposed resonator. Two varactor diodes are the attached at ends of the transmission line with tunable capacitances $C_{v 1}$ and one varactor diode with tunable capacitance $C_{v 2}$ is placed at a center point of transmission line. The DC block capacitor is placed at the center point of the transmission. For the theoretical analysis, the lossless transmission line of characteristic admittance $Y$ and physical length $L$ is assumed. Since the structure is symmetrical, the even- and odd-mode analysis method can be applied to obtain the resonant frequencies [13]. When the odd-mode excitation is applied to the ends of the proposed resonator shown in Fig. 2(a), there is a voltage null along the symmetry plane. Under odd-mode excitation, this can be represented by the half circuit, as 
shown in Fig. 2(b). Therefore, the odd-mode resonant frequency can be determined as follows:

$$
f_{\text {odd }} \times \tan \left(\frac{\pi f_{\text {odd }} L}{v_{p}}\right)=\frac{Y}{2 \pi C_{v 1}},
$$

where $v_{p}$ is the phase velocity. From (1), it is concluded that the odd-mode resonant frequency fully depends on the capacitance $C_{v 1}$ of varactor diodes connected at the ends of the transmission line. Moreover, the odd-mode resonant frequencies are not affected by the varactor diode connected at the center of the transmission line.

For even-mode excitation, there is no current flowing through the center of the transmission line. Under this condition, the proposed resonator can be represented by the equivalent half circuit shown in Fig. 2(c). For the resonance condition, the even-mode resonant frequency can be determined as follows:

$$
\left(f_{\text {even }}-\frac{Y^{2}}{4 \pi^{2} f_{\text {even }} C_{v 1} C_{v 2 \_}}\right) \tan \left(\frac{\pi f_{\text {even }} L}{v_{p}}\right)=Y \frac{C_{v 1}+C_{v 2 \_t}}{2 \pi C_{v 1} C_{v 2 \_} t},
$$

where $C_{v 2_{-} t}$ is the total capacitance for series connection of DC block capacitor and varactor diode. This is given as follows:

$$
C_{v 2_{-} t}=\frac{1}{2} \frac{C_{v 2} C_{D C}}{C_{v 2}+C_{D C}} .
$$

From (2) and (3), it can be observed that the evenmode resonant frequency depends on $C_{v 1}$ and $C_{v 2}$. Moreover, when $C_{v 1}$ is fixed, the even-mode resonant frequency can be tuned with $C_{v 2}$ alone. This characteristic of the proposed resonator can be used to design a BPF with tunable bandwidth.

To verify the theoretical analysis, a full-wave electromagnetic (EM) simulation was carried out using HFSS $v_{11}$ by Ansys. Two microstrip lines with a characteristic impedance of $50 \Omega$ were utilized to feed the proposed resonator using loose coupling to investigate its resonant behavior. The characteristic impedance of resonators is fixed at $76 \Omega$.

Fig. 3 shows the simulated resonant characteristics according to different capacitances $\left(C_{v 1}\right.$ and $\left.C_{v 2}\right)$ of the varactor diodes and length $(L)$. As $C_{v 1}$ and $C_{v 2}$ are varied, the odd- and even-mode resonant frequencies are tuned simultaneously. This characteristic of the proposed resonator can be utilized in the design of BPFs with tunable passband center frequencies. If the capacitances $C_{v 1}$ of the varactor diodes connected at the ends of the transmission line are fixed, it is obvious that the oddmode resonant frequency is fixed and the even-mode resonant frequency can be tuned by varying the capaci-
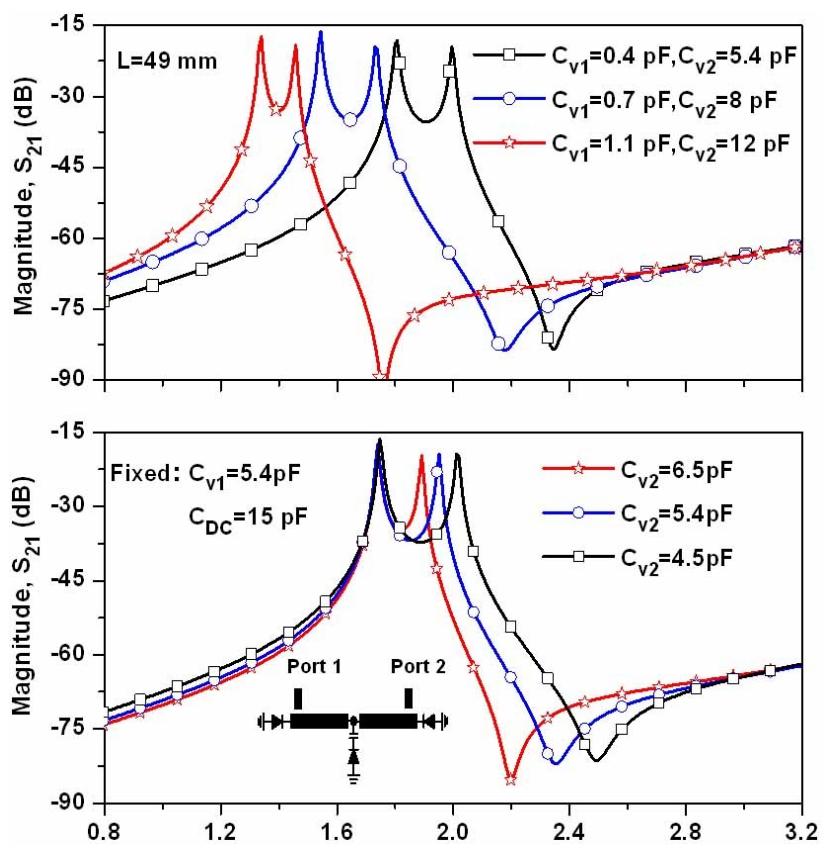

(a)
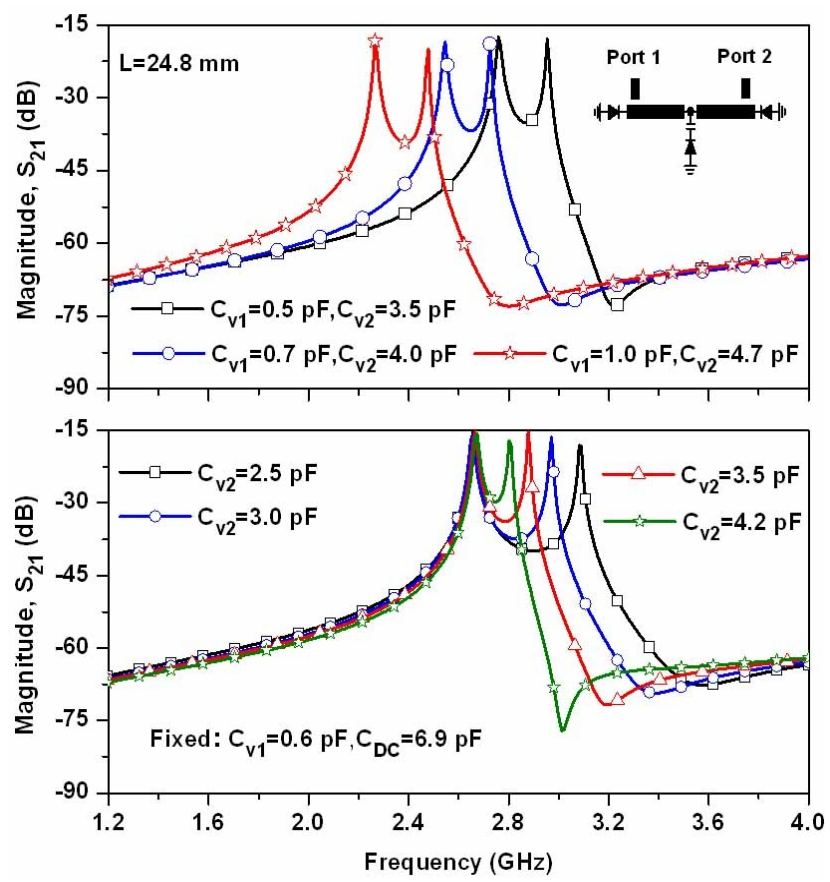

(b)

Fig. 3. Resonant frequencies of single dual-mode resonator according to capacitances and length at (a) the first passband and (b) the second passband.

tance $C_{v 2}$ of the varactor diode connected at the center of the line. The separation between the modal frequencies is proportional to the bandwidth. This characteristic of proposed resonator can be utilized in the design of BPF with tunable bandwidths.

\section{Design Method}


First, the requirement of the ideal dual-mode filter at each passband should be determined; this may be derived from its fixed frequency response centering at the high-frequency edge of a given tuning range. Then the separation of odd- and even-mode resonant frequencies and the required $Q_{\text {exo }}$ and $Q_{e x e}$ for ideal tunable filter can be determined at each passband.

Second, the tuning rate of the odd-and even-mode resonant frequencies should be determined by selecting $Y, L, C_{v 1}$, and $C_{v 2}$ using Eqs. (1) and (2) separately at each passband. Third, the coupling network should be designed to maintain the shape and bandwidth of oddand even-mode frequency responses over the tuning range.

To demonstrate the described design method, filter centerings at $f_{1}=1.80 \mathrm{GHz}$ and $f_{2}=2.9 \mathrm{GHz}$ with $3-\mathrm{dB}$ FBWs of $5.8 \%$ and $9.5 \%$ have been designed. The desired parameters can be derived from a target coupling matrix corresponding to specified response [14], which are given as $f_{\text {odd-f } 1}=1.74 \mathrm{GHz}, f_{\text {even-f } 1}=1.94 \mathrm{GHz}, Q_{\text {exo-f } 1}=$ 20.5 , and $Q_{\text {exe-fl }}=40.2$ at the lower passband $\left(f_{1}\right)$ and $f_{\text {odd } f 2}=2.76 \mathrm{GHz}, f_{\text {even-f2 }}=3.04 \mathrm{GHz}, Q_{\text {exo-f } 2}=23.5$, and $Q_{\text {exe-f2 }}$ $=29.5$ at the higher passband $\left(f_{2}\right)$. With these design parameters, the lengths and characteristic impedances of resonators at the first $\left(f_{1}\right)$ and second $\left(f_{2}\right)$ passbands are selected as $51 \mathrm{~mm}, 24.8 \mathrm{~mm}$, and $76 \Omega$, respectively. In addition, according to the external quality factors, the values of $g_{1}, g_{2}, W_{2}$, and $L_{3}$ are taken as $0.12 \mathrm{~mm}, 0.14$ $\mathrm{mm}, 0.4 \mathrm{~mm}$, and $17.5 \mathrm{~mm}$, respectively.

\section{Analysis of Center Frequency and Bandwidth Tuna- bility}

Fig. 4(a) presents the tunability of center frequency of passbands according to capacitances $C_{v 1-f 1}, C_{v 2-f 1}, C_{v 1-f 2}$, and $C_{v 2-f_{2}}$. The first passband center frequency $\left(f_{1}\right)$ can be tuned from 1.4-1.82 $\mathrm{GHz}$ by adjusting $C_{v 1-f 1}$ from 0.4 to $0.9 \mathrm{pF}$ and $C_{v 2-f 1}$ from 5.1 to $10.2 \mathrm{pF}$. Similarly, the second passband center frequency $\left(f_{2}\right)$ can be varied from 2.26 to $3 \mathrm{GHz}$ by changing $C_{v 1-f 2}$ from 0.4 to 0.9 $\mathrm{pF}$ and $C_{v 2-f 2}$ from 2.2 to $6.7 \mathrm{pF}$.

Fig. 4(b) presents the 3-dB FBWs verses capacitances (keeping $C_{v 1-f 1}$ and $C_{v 1-f 2}$ constant and varying $C_{v 2-f 1}$ and $\left.C_{v 2-f 2}\right)$ for $f_{1}=1.53-1.82$ and $f_{2}=2.38-3 \mathrm{GHz}$. From the simulation, it is found that the $3-\mathrm{dB}$ FBW results are in the tunable range of $5 \%-9 \%$ for $f_{1}=1.53-1.82 \mathrm{GHz}$ and $6 \%-12 \%$ for $f_{2}=2.38-3 \mathrm{GHz}$.

\section{Simulation and Measurement Results}

To verify the analysis of the proposed resonators, two types of tunable dual-band BPF were designed, simulated, and measured. Fig. 1(a) depicts the configuration

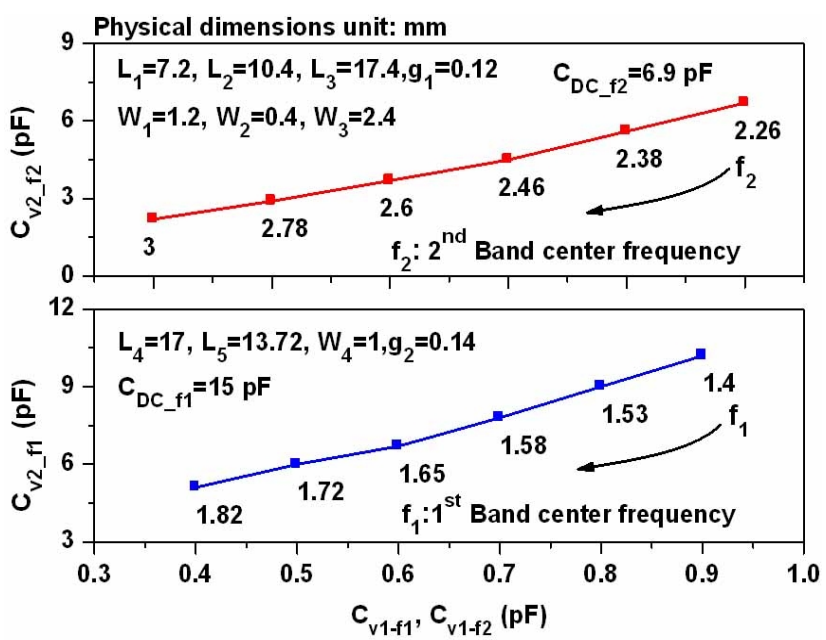

(a)
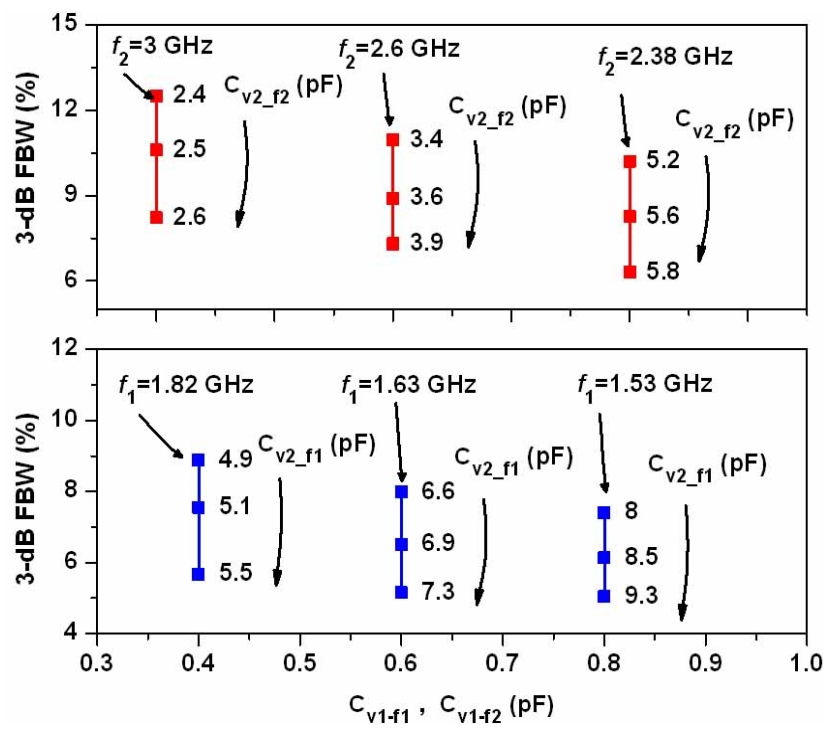

(b)

Fig. 4. Simulated results for the tunability of the center frequencies and bandwidths of passbands according to capacitances: (a) passband center frequency tunability and (b) bandwidth tunability. Refer to Fig. 1 for notation. FBW=fractional bandwidth.

of microstrip dual-mode tunable dual-band filter I. In the proposed filter, two dual-mode resonators are combined with common input/output ports. The inner resonators are designed for higher passbands, whereas the outer resonator is designed for lower passbands. The resonators are folded in order to reduce the size. The substrate is RT/Duriod 5880 made by Rogers with a dielectric constant $\left(\varepsilon_{r}\right)$ of 2.2 and thickness $(h)$ of 31 mils.

The proposed filter employs two types of varactor diodes from Skyworks: SMV1231-011LF for tuning $C_{v 1-f 1}$ and $C_{v 1-f 2}$, and SMV1233-079LF for tuning $C_{v 2-f 1}$ and $C_{v 2-f 2}$. After the simulation, the physical parameters and component values of the filter were determined as shown in Table 1. 
Table 1. Dimensions for fabricated filter I (unit $=\mathrm{mm}$; refer to Fig. 1 for notation)

\begin{tabular}{cc}
\hline$W_{1} / W_{2} / W_{3} / W_{4}$ & $1.2 / 0.4 / 2.4 / 1$ \\
$L_{1} / L_{2} / L_{3} / L_{4} / L_{5}$ & $7.2 / 10.4 / 17.4 / 17 / 13.72$ \\
$g_{1} / g_{2}$ & $0.12 / 0.14$ \\
$C_{D C f 1} / C_{D C f 2}(\mathrm{pF})$ & $15 / 6.9$ \\
\hline
\end{tabular}
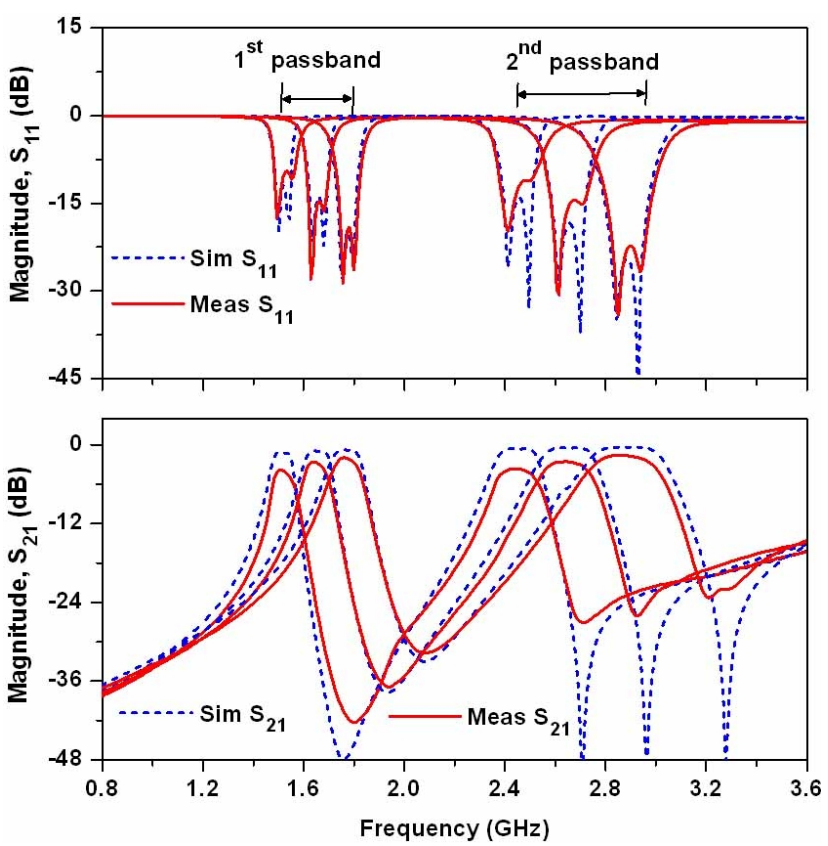

Fig. 5. Simulation (Sim) and measurement (Meas) results of filter I with both tunable passbands. Bias voltage variation: $V_{C v 1 f 1}=3.78$ to $15 \mathrm{~V}, V_{C v 2 f 1}=0.59$ to $15 \mathrm{~V}, V_{C v 1 f 2}=4$ to $15 \mathrm{~V}$, and $V_{C v 2 f 2}=4$ to $15 \mathrm{~V}$.

\section{Filter I: Tunable Center Frequencies}

Fig. 5 shows the simulation and measurement results of filter I for several typical bias voltages. The measurement results agree well with the simulation results. The measurement results show that the first passband center frequency can be tuned from 1.48 to $1.8 \mathrm{GHz}$ and the second passband center frequency can be tuned from 2.40 to $2.88 \mathrm{GHz}$.

The return loss is better than $12 \mathrm{~dB}$ in the overall tuning range of both passbands. The insertion loss varies from 1.99 to $4.4 \mathrm{~dB}$ at the first passband, whereas it varied 1.60 to $4.2 \mathrm{~dB}$ at the second passband. As the passbands are tuned towards lower frequencies, the insertion loss becomes higher. Because of decreasing bias voltages, the $\mathrm{Q}$ value of varactors becomes lower.

Fig. 6 shows the simulation and measurement results of filter I with the fixed second passband and tunable first passband center frequencies. The second passband center frequency is fixed at $2.88 \mathrm{GHz}$ and the first pass-
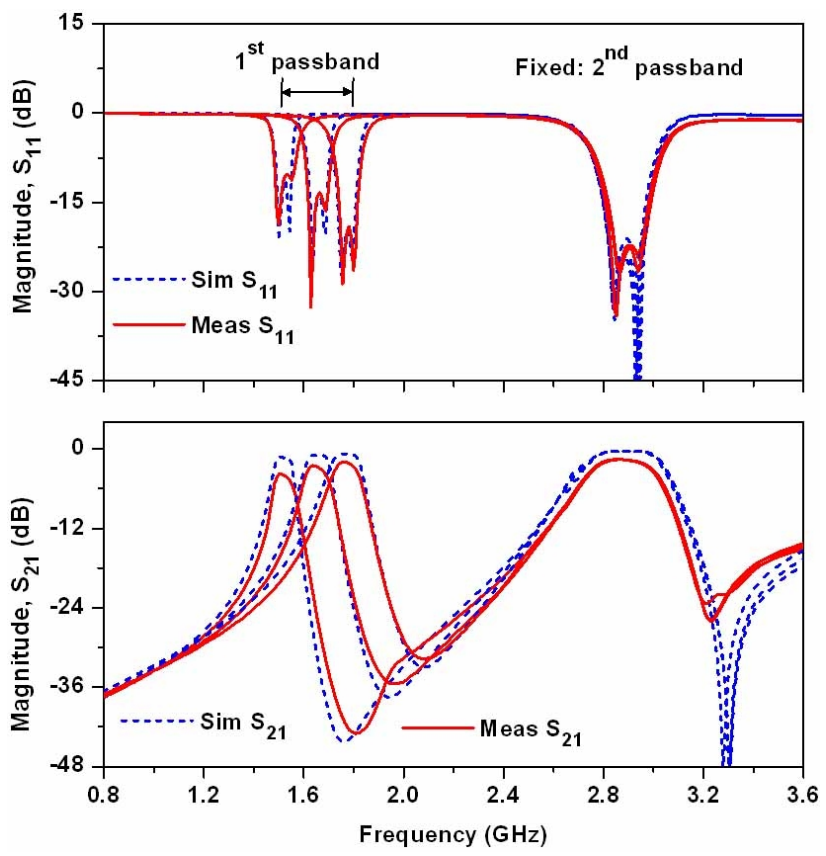

Fig. 6. Simulation and measurement results of filter I with a tunable first passband and fixed second passband. Bias voltage variation: $\mathrm{V}_{C v 1-f 1}=3.78$ to $15 \mathrm{~V}, \mathrm{~V}_{C v 2-f 1}=$ 0.59 to $15 \mathrm{~V}, \mathrm{~V}_{C v 1-f 2}=15 \mathrm{~V}$, and $\mathrm{V}_{C v 2-f 2}=15 \mathrm{~V}$.
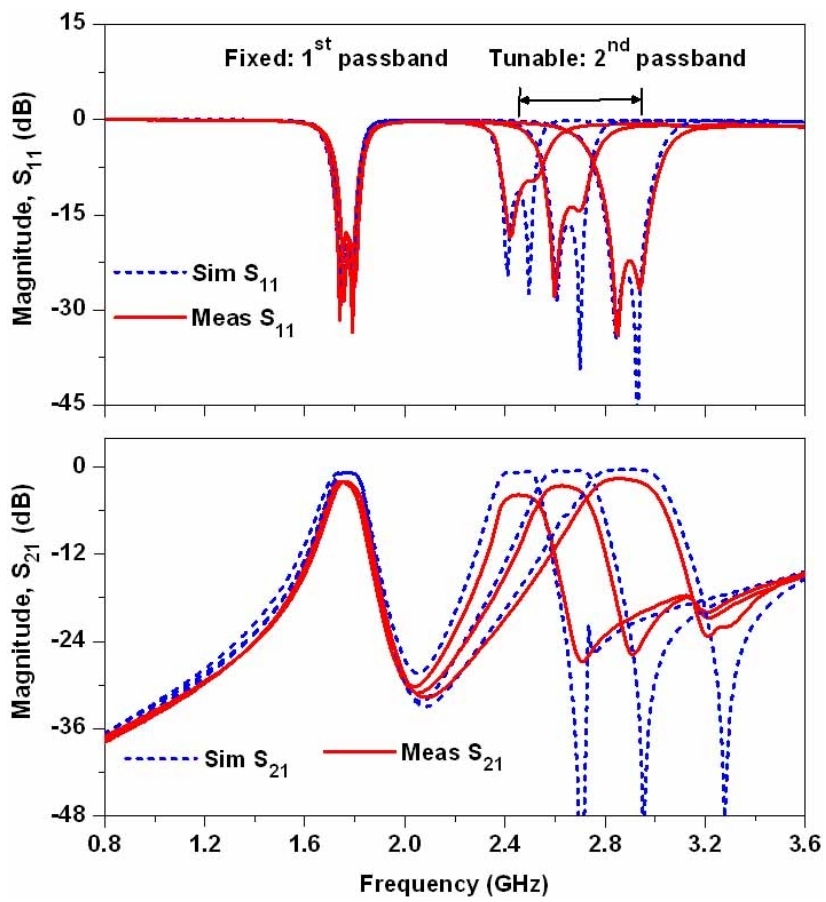

Fig. 7. Simulation and measurement results for filter I with a fixed first passband and tunable second passband. Bias voltage variation: $\mathrm{V}_{C v 1-f 1}=15 \mathrm{~V}, \mathrm{~V}_{C v 2-f 1}=15 \mathrm{~V}$, $\mathrm{V}_{C v 1-f 2}=4$ to $15 \mathrm{~V}$, and $\mathrm{V}_{C v 2-f 2}=4$ to $15 \mathrm{~V}$.

band center frequency is tuned from 1.48 to $1.8 \mathrm{GHz}$. The return loss is better than $12 \mathrm{~dB}$ over the entire tu- 
ning range of the first passband. The insertion loss varies from 1.99 to $4.42 \mathrm{~dB}$.

Fig. 7 shows the simulation and measurement results of filter I with the fixed first passband and tunable second passband center frequencies. The first passband center frequency is fixed at $1.74 \mathrm{GHz}$, and the second passband center frequency is tuned from 2.40 to 2.88 $\mathrm{GHz}$ with the return loss better than $12 \mathrm{~dB}$ over the entire tuning range.

The insertion loss varies from 1.64 to $4.2 \mathrm{~dB}$. From these results, it is clear that the center frequencies of proposed filter can be tuned independently. However, the differences in insertion loss between the simulation and measurement results are due to the use of ideal capacitances in EM simulation.

\section{Filter I: Tunable Bandwidths}

Fig. 8 shows the simulation and measurement results of filter I with both tunable passband bandwidths. As seen in this figure, the first passband's 3-dB FBWs could be tuned from $5.76 \%$ to $8.55 \%$ at $1.74 \mathrm{GHz}$ and the second passband's 3-dB FBW variation was from $8.28 \%$ to $12.42 \%$ at a center frequency of $2.80 \mathrm{GHz}$.

Figs. 9 and 10 show the simulation and measurement results for filter I, which independently verify the tunable 3-dB FBWs of the passbands. As seen in Fig. 9, the $3-\mathrm{dB}$ FBW of the first passband is tunable with the fixed second passband bandwidth. Similarly, Fig. 10 shows the simulation and measurement results for the tunable 3-dB FBWs of the second passband with the fixed first passband bandwidth. A photograph of the fabricated filter I is shown in Fig. 11.

\section{Filter II: The Tunable Dual-Band Bandpass Filter with Harmonic Suppression}

It can be observed from the simulation and measurement results in the previous section that the two passbands have several harmonics.

These harmonics are also tuned by varying the passband frequencies and must be suppressed for overall system performance.

A simple method of suppressing the harmonics is the introduction of a transmission zero at the harmonic frequency [15]. However, this method would be inefficient, because it can only suppress the harmonics around a specific frequency. Due to a broad range of variation in the harmonic frequency in the case of a tunable dual-band BPF, it is not possible to cancel harmonics sufficiently by a simple transmission zero circuit. The DGS of the microstrip line is implemented by making artificial defects on the ground plane; it provides band-re-
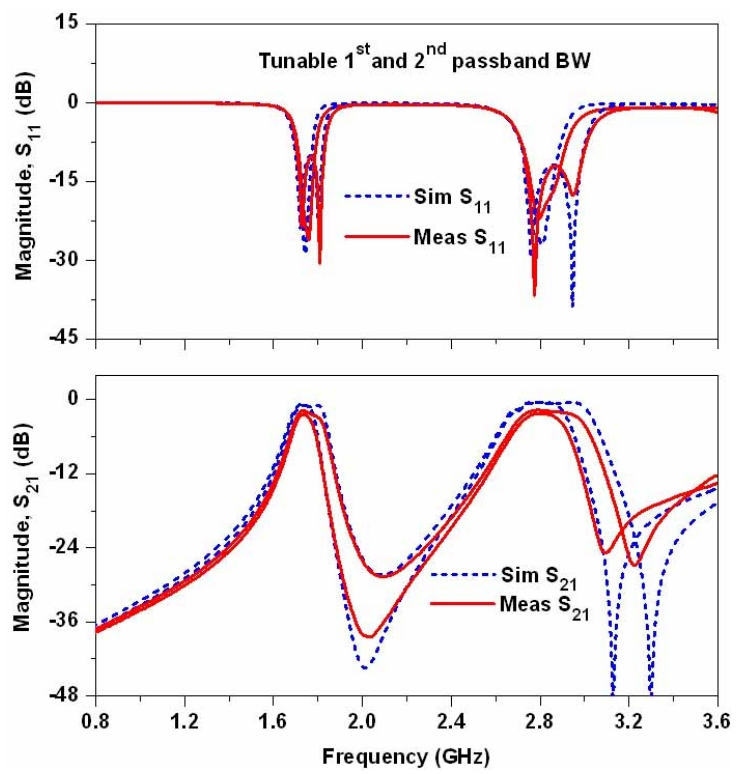

(a)

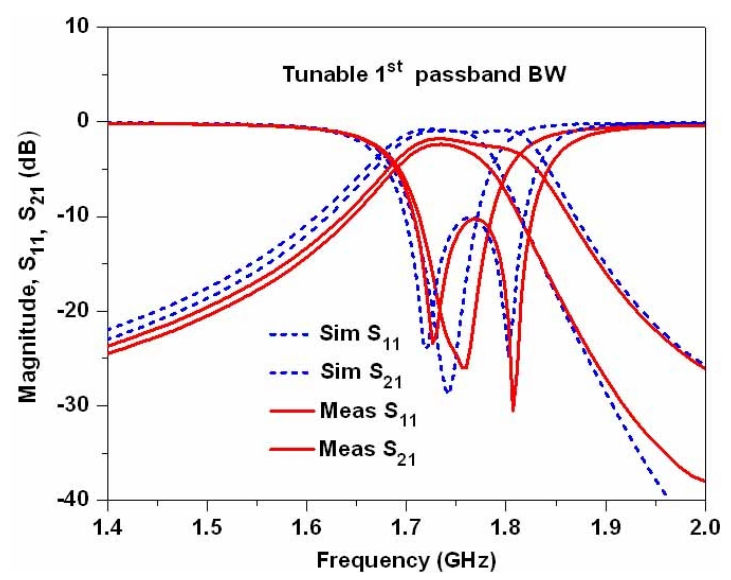

(b)

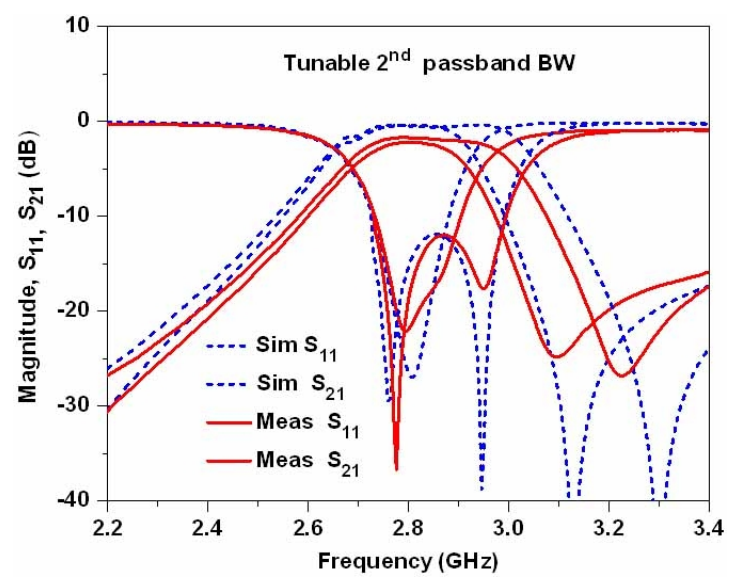

(c)

Fig. 8. Simulation and measurement results for filter I with tunable bandwidths (BWs) in both passbands simultaneously. (a) both passbands, (b) magnified first band characteristics, and (c) magnified second band characteristics. Bias voltage variation: $\mathrm{V}_{C v 1-f 1}=15 \mathrm{~V}, \mathrm{~V}_{C v 2-f 1}$ $=6$ to $15 \mathrm{~V}, \mathrm{~V}_{C \mathrm{~V} 1-\rho_{2}}=15 \mathrm{~V}$, and $\mathrm{V}_{C V 2-12}=8$ to $15 \mathrm{~V}$. 

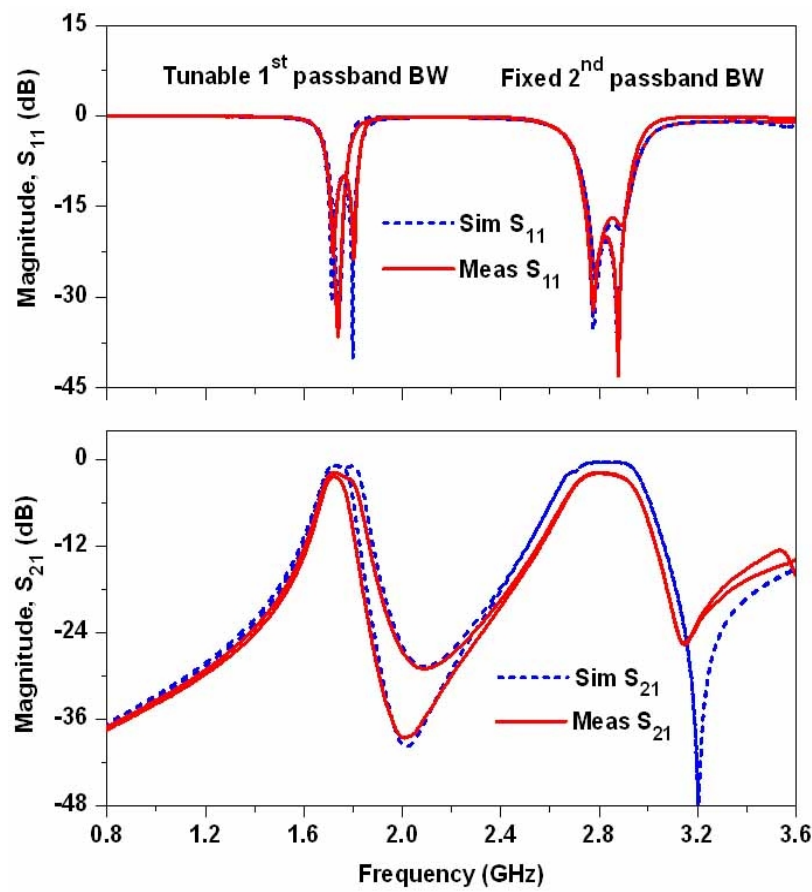

(a)

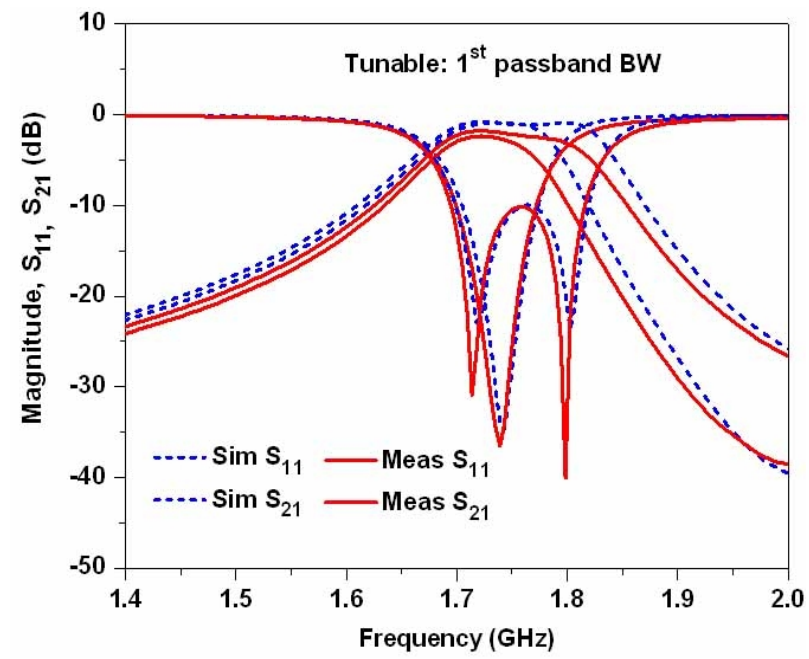

(b)

Fig. 9. Simulation and measurement results for filter I with a fixed second passband bandwidth (BW) and tunable first passband BW: (a) both passbands and (b) magnified first band characteristics. Bias voltage variation: $\mathrm{V}_{C v 1 \_1}=15 \mathrm{~V}, \mathrm{~V}_{C v 2-f 1}=6$ to $15 \mathrm{~V}, \mathrm{~V}_{C v 1-f 2}=15$ $\mathrm{V}$, and $\mathrm{V}_{C 22-\uparrow 2}=15 \mathrm{~V}$.

jection characteristics corresponding to the size and shape of defects on the ground plane. The DGS also provides an additional effective inductance in the transmission line, which enables the slow-wave factor of line to be increased. These band-rejection properties and the slow-wave effect of DGS have been applied in the design of various microwave circuits, including filters, dividers, and amplifiers [16, 17].
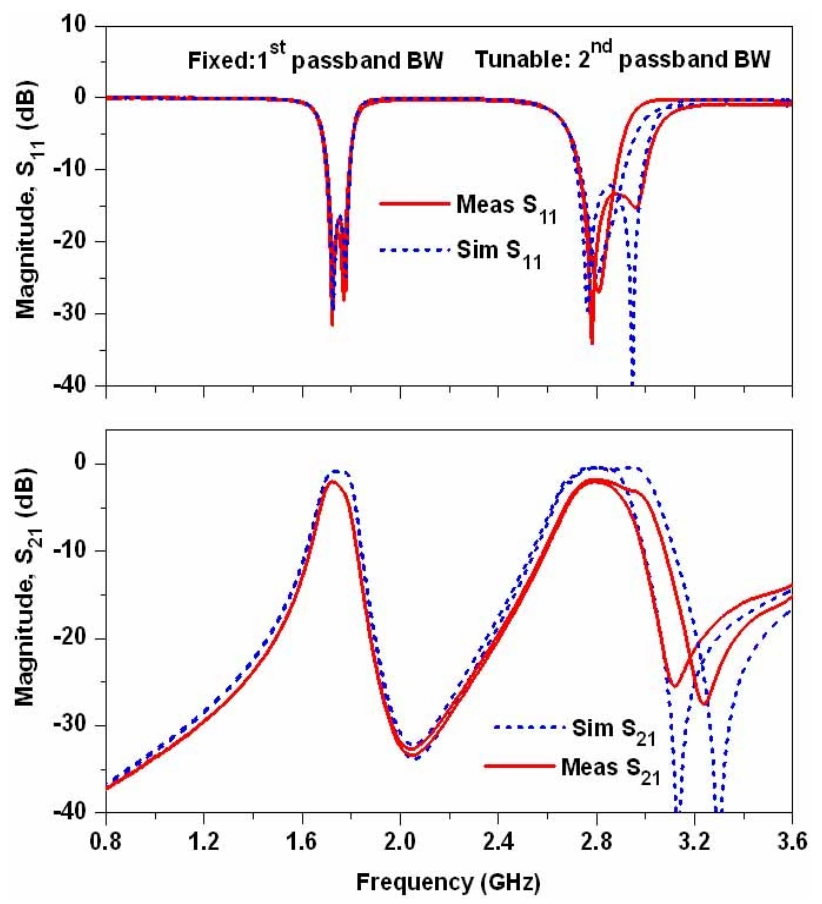

(a)

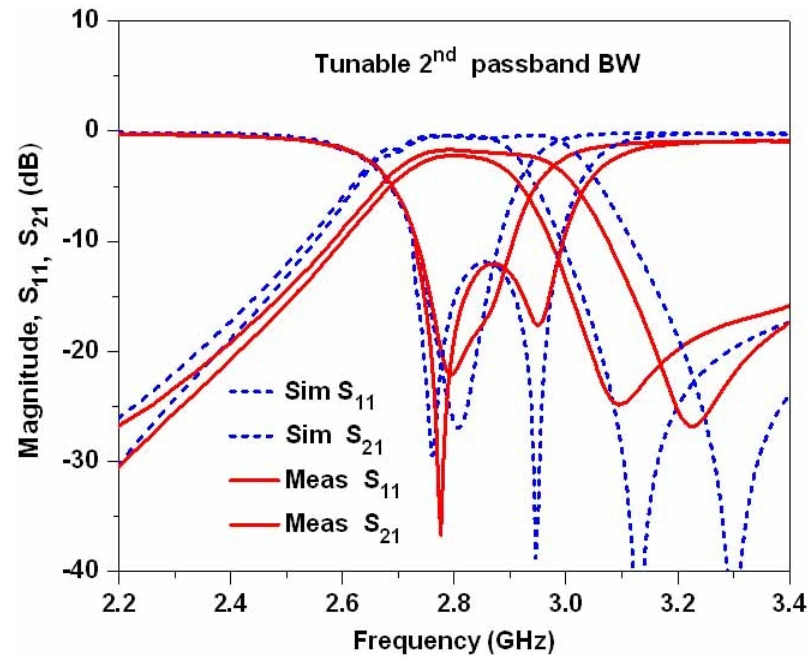

(b)

Fig. 10. Simulation and measurement results of filter I with the fixed first passband bandwidth (BW) and tunable second passband BW: (a) both passbands and (b) magnified second band characteristics. Bias voltage variation: Bias voltage variation: $\mathrm{V}_{C v 1, f 1}=15 \mathrm{~V}$, $\mathrm{V}_{C v 2-f 1}=15 \mathrm{~V}, \mathrm{~V}_{C v 1-f 2}=15 \mathrm{~V}$, and $\mathrm{V}_{C v 2-f 2}=8$ to $15 \mathrm{~V}$.

In microwave circuit design, the band-rejection property of the DGS can also be utilized in the suppression of harmonics [13, 17].

Fig. 12 shows the proposed configuration and photograph of fabricated harmonic suppressed tunable dual-band filter II. In this structure, DGS is used at the input/output feeding lines for inducting coupling and acts as a broad band-rejection resonator to suppress the 


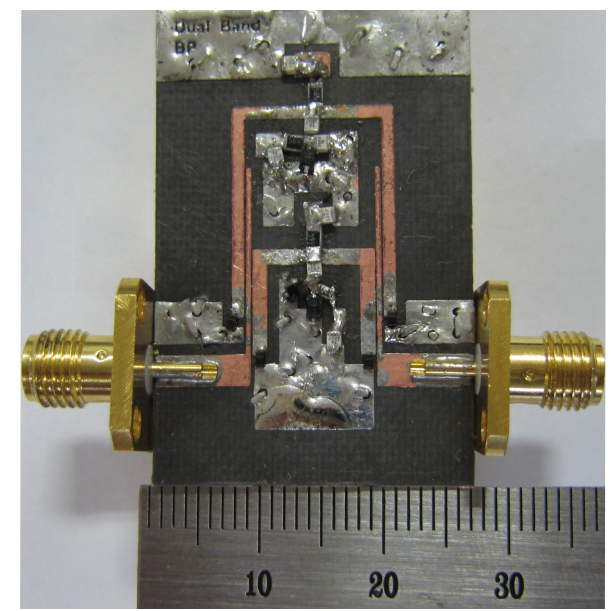

Fig. 11. Photograph of fabricated filter I.

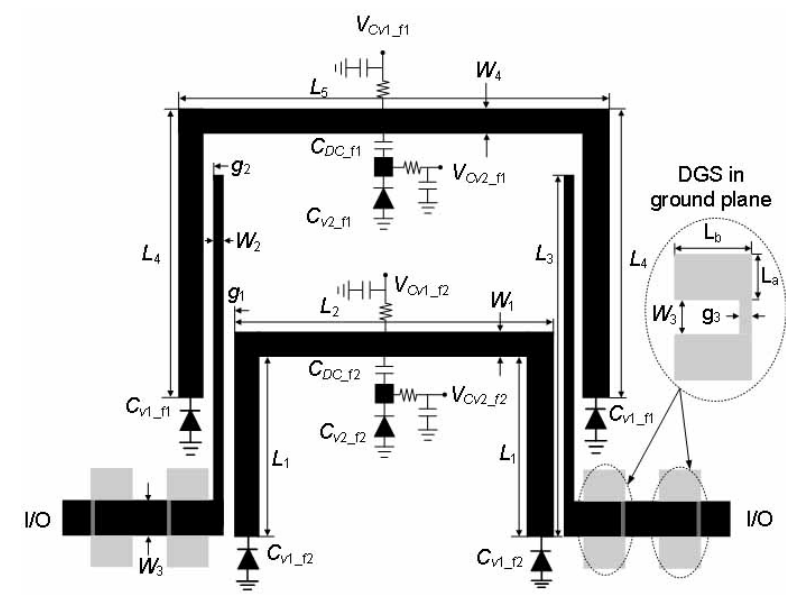

(a)

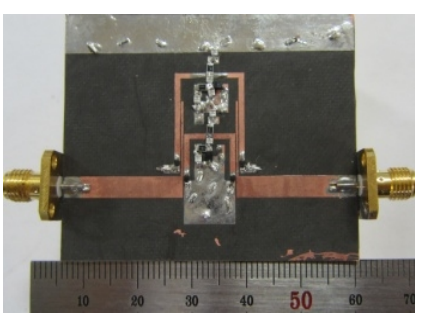

(b)

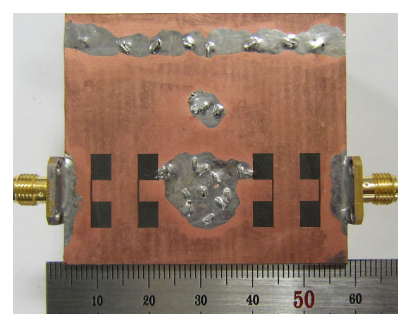

(c)
Fig. 12. Proposed structure and photograph of fabricated filter II: (a) configuration, (b) top view, and (c) bottom view of fabricated filter II. DGS=defected ground structure.

harmonics of filter I. The methods for finding the equivalent circuits of DGS are detailed in [16]. The physical parameters and component values of filter $I I$ are shown in Table 2.

Fig. 13(a) shows the narrowband characteristics of the simulation and measurement results for filter II. The passband frequencies are tuned with the help of the bias voltages of the varactor diodes. From the measurement results, the first passband frequency can be tuned from
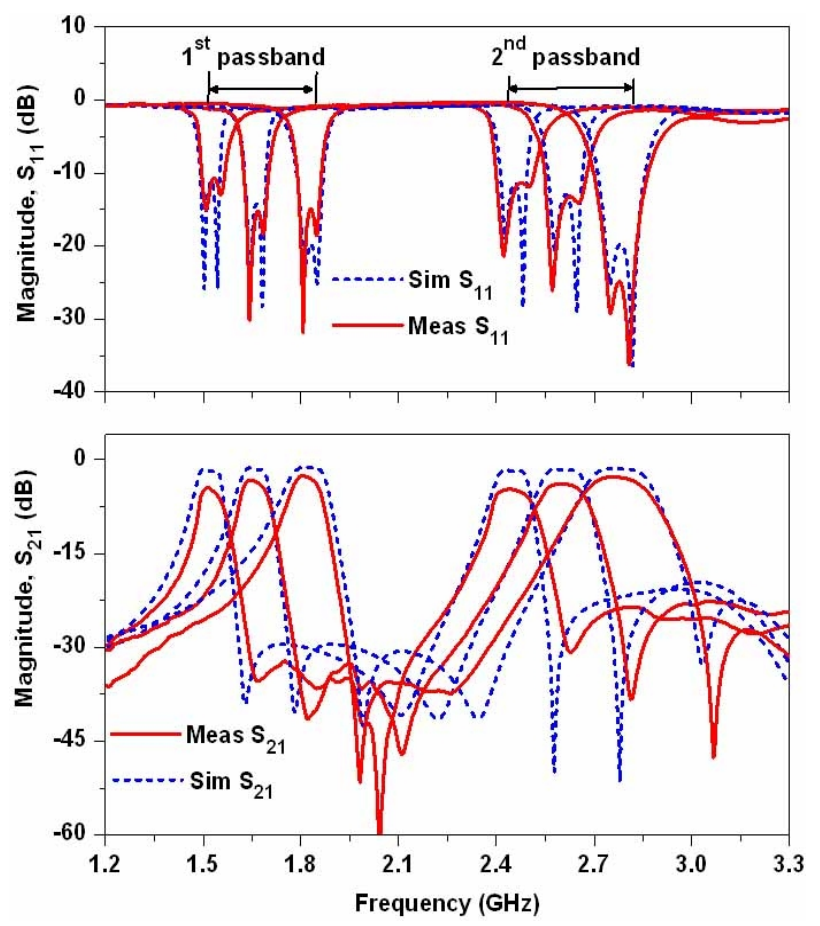

(a)

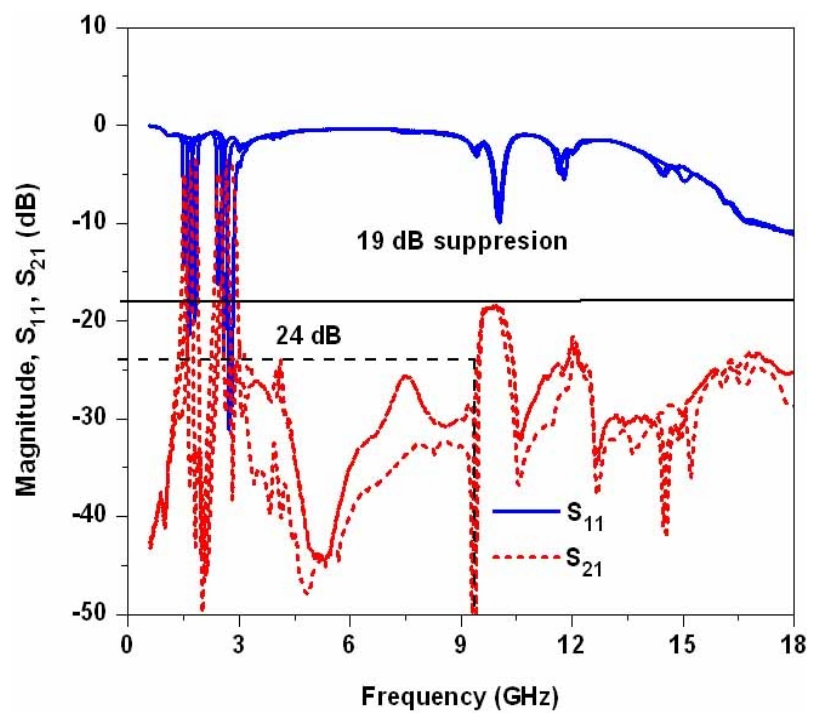

(b)

Fig. 13. Simulation and measurement results for filter II with two tunable passbands: (a) narrowband and (b) wideband characteristics. Bias voltage variation: Bias voltage variation: $\mathrm{V}_{C v 1 f 1}=4.09$ to $13 \mathrm{~V}, \mathrm{~V}_{C v 2-f 1}=0.93$ to $15 \mathrm{~V}, \mathrm{~V}_{C v 1-f 2}=5.60$ to $15 \mathrm{~V}$, and $\mathrm{V}_{C v 2-f 2}=3.21$ to $15 \mathrm{~V}$.

Table 2. Dimensions for fabricated filter II (unit=mm; refer to Fig. 12 for notation)

\begin{tabular}{cc}
\hline$W_{1} / W_{2} / W_{3} / W_{4}$ & $1.2 / 0.4 / 2.4 / 1$ \\
$L_{1} / L_{2} / L_{3} / L_{4} / L_{5} / L_{a} / L_{b}$ & $7.2 / 10.4 / 17.4 / 17 / 13.7 / 5 / 4$ \\
$g_{1} / g_{2} / g_{3}$ & $0.12 / 0.14 / 0.28$ \\
$C_{D C_{f 1} /} / C_{D C_{f 2}}(\mathrm{pF})$ & $15 / 6.9$ \\
\hline
\end{tabular}


1.5 to $1.8 \mathrm{GHz}$ and the second passband frequency can be tuned from 2.45 to $2.80 \mathrm{GHz}$. The return loss is better than $11 \mathrm{~dB}$ over the entire tuning range of the passbands. The insertion loss varies from 3.02 to $4.8 \mathrm{~dB}$ at the first passband and 2.78 to $4.6 \mathrm{~dB}$ at the second passband. These measurement results for filter II are almost similar to the filter I results in the previous section, except for the suppression of the harmonic characteristics.

In order to verify the harmonic suppression characteristics of filter $I$, the broadband harmonic suppression characteristics are shown in Fig. 13(b). The harmonic suppression characteristics for the fabricated filter II are better than $19 \mathrm{~dB}$ up to $18 \mathrm{GHz}$ over the entire tuning range of the passbands. This means that the proposed structure can suppress more than 10th order harmonics of the highest center frequencies of the first passbands due to the band-rejection characteristics of DGS. This confirmed that the proposed method can achieve broadband harmonic suppression without degrading any passbands performances. Performance comparisons of the proposed tunable filter with other tunable filters reported in the literature are summarized in Table 3 . The proposed filter can provide dual-passbands with independently tunable center frequencies and bandwidths in ad-

Table 3. Performance comparison of tunable filter with independent control of center frequency and bandwidth

\begin{tabular}{|c|c|c|c|c|c|c|}
\hline \multirow[t]{2}{*}{ Ref. } & \multicolumn{2}{|c|}{$\begin{array}{l}\text { Frequency ten- } \\
\text { ability (GHz) }\end{array}$} & \multicolumn{2}{|c|}{$\begin{array}{l}\text { 3-dB FBW ten- } \\
\text { ability }(\%)\end{array}$} & \multirow[t]{2}{*}{ A } & \multirow{2}{*}{$\begin{array}{l}\text { Harmonic sup- } \\
\text { pression order }\end{array}$} \\
\hline & $f_{1}$ & $f_{2}$ & $f_{1}$ & $f_{2}$ & & \\
\hline$[5]$ & $\begin{array}{l}0.85- \\
1.40\end{array}$ & - & $\begin{array}{l}2.90- \\
5.20^{*}\end{array}$ & - & $\mathrm{N}$ & - \\
\hline [8] & $\begin{array}{l}1.50- \\
2.20\end{array}$ & - & $\begin{array}{c}2.20- \\
12.20^{*}\end{array}$ & - & $\mathrm{Y}$ & - \\
\hline [9] & $\begin{array}{l}1.75- \\
2.25\end{array}$ & - & $\begin{array}{l}4.00- \\
5.71^{*}\end{array}$ & - & $\mathrm{Y}$ & - \\
\hline [10] & $\begin{array}{l}2.90- \\
3.50\end{array}$ & - & $\begin{array}{l}4.00- \\
12.00\end{array}$ & - & $\mathrm{Y}$ & - \\
\hline [11] & $\begin{array}{c}3.70- \\
6.00 * *\end{array}$ & - & $\begin{array}{l}1.60- \\
9.30^{*}\end{array}$ & - & $\mathrm{Y}$ & - \\
\hline [12] & $\begin{array}{l}2.20- \\
2.70\end{array}$ & $\begin{array}{l}3.45- \\
4.20\end{array}$ & - & - & $\mathrm{N}$ & $\begin{array}{l}-20 \mathrm{~dB} \text { up to } \\
1.75 f_{2_{2} \max }\end{array}$ \\
\hline [13] & $\begin{array}{l}0.85- \\
1.20\end{array}$ & $\begin{array}{l}1.40- \\
2.14\end{array}$ & - & - & $\mathrm{N}$ & $\begin{array}{l}-20 \mathrm{~dB} \text { more } \\
\text { than } 10 f_{2 \_ \text {max }}\end{array}$ \\
\hline $\begin{array}{l}\text { This } \\
\text { work }\end{array}$ & $\begin{array}{l}1.48- \\
1.80\end{array}$ & $\begin{array}{l}2.40- \\
2.88\end{array}$ & $\begin{array}{l}5.70- \\
8.50\end{array}$ & $\begin{array}{l}8.20- \\
12.40\end{array}$ & $\mathrm{Y}$ & $\begin{array}{l}-19 \mathrm{~dB} \text { more } \\
\text { than } 10 f_{1 \_\max }\end{array}$ \\
\hline
\end{tabular}

$\mathrm{FBW}=$ fractional bandwidth, $\mathrm{A}=$ independently tunable center frequency and bandwidth, $\mathrm{Y}=\mathrm{yes}, \mathrm{N}=\mathrm{no}, f_{1 \_ \text {max }}=$ highest center frequency of first passband, $f_{2 \_ \text {max }}=$ highest center frequency of second passband, $*=1-\mathrm{dB} \mathrm{FBW}, * *=$ suspended-stripline rings in micro-electromechanical system technology. dition to broadband harmonic rejection characteristics.

\section{Conclusion}

In this paper, the design of tunable dual-band BFPs with independently tunable center frequencies and bandwidths was demonstrated. The proposed structure is validated by both theoretical analysis and experiments. Defected ground structures were utilized to reject the harmonics. The experimental results are in good agreement with the theoretical predictions. These experimental results showed that the first passband can be tuned from 1.48 to $1.8 \mathrm{GHz}$ with a $3-\mathrm{dB}$ fractional bandwidth tunability of $5.76 \%$ to $8.55 \%$, while the second passband can be tuned from 2.40 to $2.88 \mathrm{GHz}$ with a $3-\mathrm{dB}$ fractional bandwidth tunability of $8.25 \%$ to $12.42 \%$. The proposed filter also offers switchable passband characteristics.

The proposed method can suppress more than 10th order harmonics of second passbands, thereby ensuring broad harmonic rejection characteristics without any degradation of the passband characteristics. The proposed filter topology is simple to implement and a good candidate for communication systems requiring multiband tunable filters with center frequency and bandwidth control. The proposed filter design method can be applied to selectable multimode or multiband applications that have different operating frequencies and bandwidths.

\section{References}

[1] K. Entesari and G. M. Rebeiz, "A differential 4-bit 6.5 -10-GHz RF MEMS tunable filter," IEEE Transactions on Microwave Theory and Techniques, vol. 53, no. 3, pp. 1103-1110, Mar. 2005.

[2] S. Park, M. A. El-Tanani, I. Reines, and G. M. Rebeiz, "Low-loss 4-6-GHz tunable filter with 3-bit high-Q orthogonal bias RF-MEMS capacitance network," IEEE Transactions on Microwave Theory and Techniques, vol. 56, no. 10, pp. 2348-2355, Oct. 2008.

[3] J. Nath, D. Ghosh, J. P. Maria, A. I. Kingon, W. Fathelbab, P. D. Franzon, and M. B. Steer, "An electronically tunable microstrip bandpass filter using thinfilm barium-strontium-titanate (BST) varactors," IEEE Transactions on Microwave Theory and Techniques, vol. 53, no. 9, pp. 2707-2712, Sep. 2005.

[4] B. Liu, F. Wei, and X. Shi, "Reconfigurable bandpass filter based on net-type stepped-impedance resonator," Electronics Letters, vol. 46, no. 22, pp. 15061507, Oct. 2010.

[5] S. J. Park and G. M. Rebeiz, "Low-loss two-pole 
tunable filters with three different predefined bandwidth characteristics," IEEE Transactions on Microwave Theory and Techniques, vol. 56, no. 5, pp. 1137-1148, May 2008.

[6] W. Tang and J. Hong, "Varactor-tuned dual-mode bandpass filters," IEEE Transactions on Microwave Theory and Techniques, vol. 58, no. 8, pp. 22132219, Aug. 2010.

[7] J. Long, C. Li, W. Cui, J. Huangfu, and L. Ran, "A tunable microstrip bandpass filter with two independently adjustable transmission zeros," IEEE Microwave and Wireless Components Letters, vol. 21, no. 2, pp. 74-76, Feb. 2011.

[8] Y. Chiou and G. M. Rebeiz, "A tunable three-pole 1.5-2.2-GHz bandpass filter with bandwidth and transmission zero control," IEEE Transactions on Microwave Theory and Techniques, vol. 59, no. 11, pp. 2872-2878, Nov. 2011.

[9] Y. Chiou and G. M. Rebeiz, "A quasi elliptic function 1.75-2.25 GHz 3-pole bandpass filter with bandwidth control," IEEE Transactions on Microwave Theory and Techniques, vol. 60, no. 2, pp. 244-249, Feb. 2012.

[10] A. L. C. Serrano, F. S. Correra, T. P. Vuong, and P. Ferrari, "Synthesis methodology applied to a tunable patch filter with independent frequency and bandwidth control," IEEE Transactions on Microwave Theory and Techniques, vol. 60, no. 3, pp. 484-493, Mar. 2012.

[11] C. C. Cheng and G. M. Rebeiz, "High-Q 4-6-GHz suspended stripline RF MEMS tunable filter with bandwidth control," IEEE Transactions on Microwave Theory and Techniques, vol. 59, no. 10, pp.
2469-2476, Oct. 2011.

[12] E. E. Djoumessi, M. Chaker, and K. Wu, "Varactor-tuned quarter-wavelength dual-bandpass filter," IET Microwaves, Antennas and Propagation, vol. 3, no. 1, pp. 117-124, Feb. 2009.

[13] G. Chaudhary, Y. Jeong, and J. Lim, "Harmonic suppressed dual-band bandpass filters with tunable passbands," IEEE Transactions on Microwave Theory and Techniques, vol. 60, no. 7, pp. 21152123, Jul. 2012.

[14] S. Amari, U. Rosenberg, and J. Bornemann, "Adaptive synthesis and design of resonator filters with source/load-multi-resonator coupling," IEEE Transactions on Microwave Theory and Techniques, vol. 50, no. 8, pp. 1969-1978, Aug. 2002.

[15] S. Sun and L. Zhu, "Periodically nonuniform coupled microstrip-line filters with harmonic suppression using transmission zero reallocation," IEEE Transactions on Microwave Theory and Techniques, vol. 53, no. 5. pp. 1817-1822, May 2005.

[16] D. Ahn, J. S. Park, C. S. Kim, J. N. Kim, Y. Qian, and $\mathrm{T}$. Itoh, "A design of low-pass filter using the novel microstrip defected ground structure," IEEE Transactions on Microwave Theory and Techniques, vol. 49, no. 1, pp. 86-93, Jan. 2001.

[17] C. S. Kim, D. H. Kim, I. S. Song, K. M. K. H. Leong, T. Itoh, and D. Ahn, "A design of a ring bandpass filters with wide rejection band using DGS and spur-line coupling structures," presented at 2005 IEEE MTT-S International Microwave Symposium Digest, Long Beach, CA, Jun. 12-17, 2005, pp. 2183-2186. 


\section{Girdhari Chaudhary}

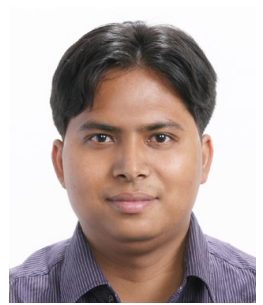

received the B.E. and M. Tech degrees in Electronics and Communication Engineering from Nepal Engineering College (NEC), Kathmandu, Nepal and Malaviya National Institute of Technology (MNIT), Jaipur, India in 2004 and 2007, respectively and Ph.D. degree in Electronics Engineering from Chonbuk National University, Jeonju, Republic of Korea in 2013. Now, he is working toward Postdoc researcher in Chonbuk National University. His research interests include multi-band passive circuits, negative group delay filters, and high efficiency power amplifiers. He is a student member of IEEE and Korean Institute of Electromagnetic Engineering and Science (KIEES).
Yongchae Jeong

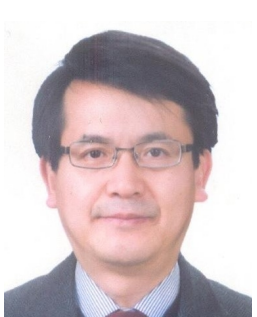

received B.S.E.E. and M.S.E.E., and $\mathrm{Ph}$. D. degrees in electronics engineering from Sogang University, Seoul, Republic of Korea in 1989 and 1991, respectively. From 1991 to 1998 , he worked as a senior engineer with Samsung Electronics. From 1998, he joined division of electronic engineering, Chonbuk National University, Jeonju, Republic of Korea. From July 2006 to December 2007, he joined at Georgia Institute of Technology as a visiting professor. He was a recipient of Distinguished Research Award of KIEES (Korea Institute of Electromagnetic Engineering and Science) in 2008. Now, he is a professor and member of IT convergence research center in Chonbuk National Univeristy. He is currently teaching and conducting research in the area of $\mathrm{RF} /$ microwave periodic transmission structure, MIC/MMIC passive and active circuit design for the mobile and satellite communication, specializing in base station amplifiers, nonlinear devices and system linerizing technology, and RFIC design. He is a senior member of IEEE and member of KIEES. He has authored and co-authored over 100 papers in international journals and conference proceedings.
Jongsik Lim

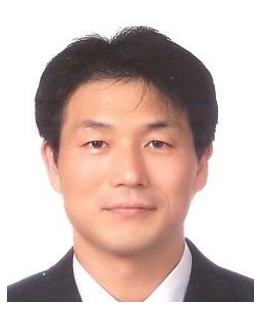

received the B.S. and M.S. in electronic engineering from Sogang University, Seoul, Republic of Korea, in 1991 and 1993, and Ph.D. degree from the school of electrical engineering and computer science, Seoul National University in 2003. In 1993, he joined ETRI, Daejeon, Republic of Korea and was with them for 6 years in Satellite Communication division as a senior member of research statff. He was one of the key members in developing MMIC LNA and SSPA for $20 / 30 \mathrm{GHz}$ satellite transponder in ETRI. From March to July in 2003, he worked in Division of Information Technology, Brain Korea 21 Project in Seoul National University as a post doctoral fellow, and gave lectures in the graduate schools of Soonchunhyang University and Soongsil University. He has experiences as a patent examiner in the Korean Intellectual Property Office (KIPO) through July 2003 to September 2004. In September 2004, he joined ETRI again and worked in the Antenna Technology Research Team/Radio Technology Group as a senior research member. Since March 2005, he has been with the Department of Electrical and Communication Engineering, Soonchunhyang University in Korea as a faculty member. His current research interests include design of the passive and active circuits for $\mathrm{RF} /$ microwave and millimeter-wave with MIC/MMIC technology, modeling of active device, design of high power amplifiers for mobile communications, applications of periodic structure to the $\mathrm{RF} /$ microwave circuits and modeling of passive structure having periodic structures. He is a member of IEEE, IEICE and KIEES (Korea Institute of Electromagnetic Engineering and Science). 